\title{
Genomewide association study identifies no major founder variant in Caucasian moyamoya disease
}

\author{
WANYANG LIU ${ }^{1,7}$, S. T. M. L. D. SENEVIRATHNA ${ }^{1}$, TOSHIAKI HITOMI ${ }^{1}$, HATASU KOBAYASHI ${ }^{1}$, \\ CONSTANTIN RODER ${ }^{2}$, ROMAN HERZIG ${ }^{3}$, MARKUS KRAEMER ${ }^{4}$, MAURITS H. J. VOORMOLEN ${ }^{5}$, \\ PAVLÍNA CAHOVÁ ${ }^{6}$, BORIS KRISCHEK ${ }^{2 *}$ and AKIO KOIZUMI ${ }^{1 *}$
}

\author{
${ }^{1}$ Department of Health and Environmental Sciences, Kyoto University Graduate School of Medicine, Kyoto 606-8501, Japan \\ ${ }^{2}$ Department of Neurosurgery, University of Tübingen, Tübingen D-72076, Germany \\ ${ }^{3}$ Stroke Comprehensive Center, Department of Neurology, Faculty of Medicine and Dentistry, \\ Palacký University and University Hospital, Olomouc 77900, Czech Republic \\ ${ }^{4}$ Department of Neurology, Alfried-Krupp-von Bohlen und Halbach Hospital, Essen 45117, Germany \\ ${ }^{5}$ Department of Radiology, Antwerp University Hospital and University of Antwerp, Edegem BE-2000, Belgium \\ ${ }^{6}$ Department of Child Neurology, University Hospital, Brno 62500, Czech Republic \\ ${ }^{7}$ Department of Nutrition and Food Hygiene, School of Public Health, China Medical University, \\ Shenyang 110001, People's Republic of China
}

\begin{abstract}
[Liu W., Senevirathna S. T. M. L. D., Hitomi T., Kobayashi H., Roder C., Herzig R., Kraemer M., Voormolen M. H. J., Cahová P., Krischek B. and Koizumi A. 2013 Genomewide association study identifies no major founder variant in Caucasian moyamoya disease. J. Genet. 92, $\mathrm{xx}-\mathrm{xx}]$
\end{abstract}

\section{Introduction}

Moyamoya disease (MMD) is an idiopathic cerebrovascular occlusive-stenosis disorder at the terminal portion of internal carotid arteries and its main branches, accompanied by collateral vascular networks at the base of the circle of Willis (Takeuchi and Shimizu 1957; Suzuki and Takaku 1969). MMD has the highest prevalence in East Asian countries and a low prevalence in European countries (Goto and Yonekawa 1992; Kuroda and Houkin 2008). We have found that the p.R4810K variant in the ring finger protein 213 (RNF213) is a major founder susceptibility gene for East Asian MMD (Liu et al. 2010, 2011). In this study, we aimed to test whether there is a major founder susceptibility gene for Caucasian MMD using a genomewide association study (GWAS). We demonstrated that there was no major founder variant in Caucasian MMD as it is in East Asian MMD. We identified several suggestive association regions for Caucasian MMD.

\footnotetext{
*For correspondence. E-mail: Boris Krischek, krischek@gmail.com; Akio Koizumi, koizumi.akio.5v@kyoto-u.ac.jp.

Wanyang Liu and S. T. M. L. D. Senevirathna contributed equally to this work.
}

\section{Materials and methods}

\section{Study subjects}

Ethical approval was given by the Institutional Review Boards and Ethics Committees of Kyoto University School of Medicine, Japan; Medical Faculty of the University of Tübingen, Germany; and the Faculty of Medicine and Dentistry of Palacký University, Czech Republic, respectively. Written informed consent was obtained from all participants. We calculated the required size of the study population to obtain enough statistical power in Caucasian population by assuming the following conditions as was reported in East Asian population (Liu et al. 2011): the frequency of the high risk allele is 0.01 , the prevalence of MMD is $<10^{-4}$; the relative risk of risk allele is 100 by assuming an autosomal dominant mode of inheritance; the marker $D^{\prime}$ is 0.99 and its frequency $>0.05$; type I error is 0.05 and statistical power $\geq 0.8$ (http://pngu.mgh.harvard.edu/ purcell/gpc/). The calculation indicated that 40 for cases and controls can assure the statistical power of 0.8. Cases are composed of 38 unrelated subjects: seven were Czech and 31 were German. Those cases were case-series patients between February 2008 and November 2009 in participating hospitals. Controls were all unrelated Germans, in whom, absence of MMD were confirmed by magnetic resonance imaging (MRI) in the same period.

Keywords. Caucasian; founder variant; genomewide association study; moyamoya disease; suggestive association. 
Genomic DNA was extracted from the blood samples using a QIAamp DNA Blood Mini Kit (Qiagen, Germantown, USA) according to the manufacturer's protocol. Genomewide genotyping was performed using an Illumina Human610Quad BeadChip Kit (Illumina, San Diego, USA). Samples with overall call rates $<95 \%$ were excluded. Single nucleotide polymorphisms (SNPs) that met the following criteria were excluded: had call rates $<0.99$ in all GWAS samples and genotype distributions deviated from those expected by Hardy-Weinberg equilibrium (HWE). Principal component analysis showed no outliers or population stratification, and all subjects were confirmed to be of Caucasian ethnicity (see figure 1 in electronic supplementary material at http://www. ias.ac.in/jgenet/). A quantile-quantile plot revealed that the distribution of observed $P$ values followed the expected distribution (see figure 2 in electronic supplementary material). After quality control, 565,294 out of 620,901 SNPs for the 38 cases and 41 controls were retained for GWAS.

\section{Candidate genes sequencing by the Sanger method}

Five cases and one control were randomly selected for sequencing of all coding exons and at least 100-bp exonintronic boundaries in eight candidate genes from within five suggestive association regions for the case-control study. The primers that were used for direct sequencing are shown in table 1 in electronic supplementary material. The sequencing products were separated on an ABI Prism 3100 Avant DNA sequencer (Applied Biosystems, Kyoto, Japan). In addition, we investigated the association of SNPs in known two genes, actin, alpha 2, smooth muscle, aorta (ACTA2) (Guo et al. 2009) and RNF213 (Liu et al. 2011), with MMD by GWAS.

\section{Statistical analysis}

GWAS was conducted using the SNP and Variation Suite V7 (Golden Helix, Bozeman, USA). Thresholds of $5 \times 10^{-8}$ and $1 \times 10^{-5}$ were set for genomewide significance and suggestive association, respectively. We assumed that a minor allele frequency of the SNP (i.e., risk marker allele), which is postulated to be the closest marker of the putative moyamoya susceptibility gene, was 0.5 in cases and 0.05 in controls as it does in Japanese population (Liu et al. 2011). Under this condition, with the current population sizes for cases and controls, simulation (http://pngu.mgh.harvard.edu/ purcell/gpc/) predicts that 29 out of 38 cases are homozygous or heterozygous for the risk marker allele while four controls were heterozygous for the risk marker allele and 37 controls were homozygous for the wild marker allele. Given an autosomal dominant in HWE, we can expect the odds ratio of the risk marker allele to be 29.8 with $P=9.53 \times 10^{-10}$. When we assume a more modest condition for an odds ratio of 5 , the statistical power was $2 \times 10^{-3}$ and 215 cases and controls are needed to obtain the SNPs with the genomewide significant $P$ of $5 \times 10^{-8}$.

\section{Results}

\section{Demographic and clinical profiles of the participants}

The participants comprised 38 cases and 41 unrelated controls (see table 2 in electronic supplementary material). The mean age for cases and controls was $31.37 \pm 14.83$ and $27.34 \pm 11.55$ years, respectively. The sex ratio of male:female was 14:24 and 8:33 for cases and controls, respectively. Age of onset for the cases was $22.93 \pm 16.95$ years which indicates that the adult onset was dominant. Cerebral infarction was the major clinical symptom, followed by transient ischemic attack and others. Thirty-five of the cases were diagnosed as bilateral MMD; the other three were unilateral MMD.

\section{Genomewide association study}

GWAS was performed under two conditions. One was case-control comparison and the other was cases with and without cerebral infarction comparison. No SNPs reached genomewide significant association under both conditions $\left(P<5 \times 10^{-8}\right.$, figure 1). However, GWAS revealed multiple suggestive associations under case-control comparison $(P<$ $1 \times 10^{-5}$, figures $1 \mathrm{a}$ and $\left.2 \mathrm{a}-\mathrm{d}\right)$. The top association SNPs were rs1023115 (odds ratio, $\mathrm{OR}=6.63, P=2.60 \times 10^{-6}$ ) on $1 \mathrm{q} 23.3, \quad \mathrm{rs} 11681583 \quad\left(\mathrm{OR}=6.51, \quad P=2.93 \times 10^{-6}\right)$ on $2 \mathrm{p} 22.1, \operatorname{rs} 3742257\left(\mathrm{OR}=0.20, P=5.73 \times 10^{-6}\right)$ on 13q14.11, rs2058364 $\left(\mathrm{OR}=5.57, P=5.90 \times 10^{-6}\right) \quad$ on 17 p13.3 and rs720607 (OR $\left.=0.17, P=5.59 \times 10^{-6}\right)$ on 20 q13.33 (see table 3 in electronic supplementary material; figure 2, a-d). Under case-only analysis, two SNPs reached genomewide suggestive associations (rs17078141 on 3p22.1, $\mathrm{OR}=0.04, P=6.10 \times 10^{-6}$; rs2178763 on $4 \mathrm{q} 22.3$, OR $=$ $21.81, P=6.88 \times 10^{-6}$, table 3 in electronic supplementary material; figures $1 \mathrm{~b}$ and $2 \mathrm{e}$ ).

\section{Mutation analysis of candidate genes}

Candidate regions covering top suggestive association SNPs were further investigated by direct sequencing (figure 2). There was no annotated gene on $17 \mathrm{p} 13.3$ and $4 \mathrm{q} 22.3$ that covered rs2058364 and rs2178763, respectively. A summary of the variants detected by direct sequencing is available in table 4 in electronic supplementary material for the casecontrol study. We identified a total of 79 variants among which five were missense and eight were synonymous; the remaining variants were located in noncoding regions. We considered that synonymous and noncoding variants were unlikely to cause the MMD, even when they have low frequencies in general population. All of the missense variants detected were found in dbSNPs database (http://www.ncbi. nlm.nih.gov/projects/SNP/, NCBI build 36.3). Their allele frequencies in general Caucasian populations were greater than 0.1, suggesting that these missense SNPs were unlikely to be associated with the development of MMD in the Caucasian population. In terms of case-only analysis, four genes (MYRIP, FLJ33065, ENTPD3 and FLJ36665) were found but no sequence determinations were done. 

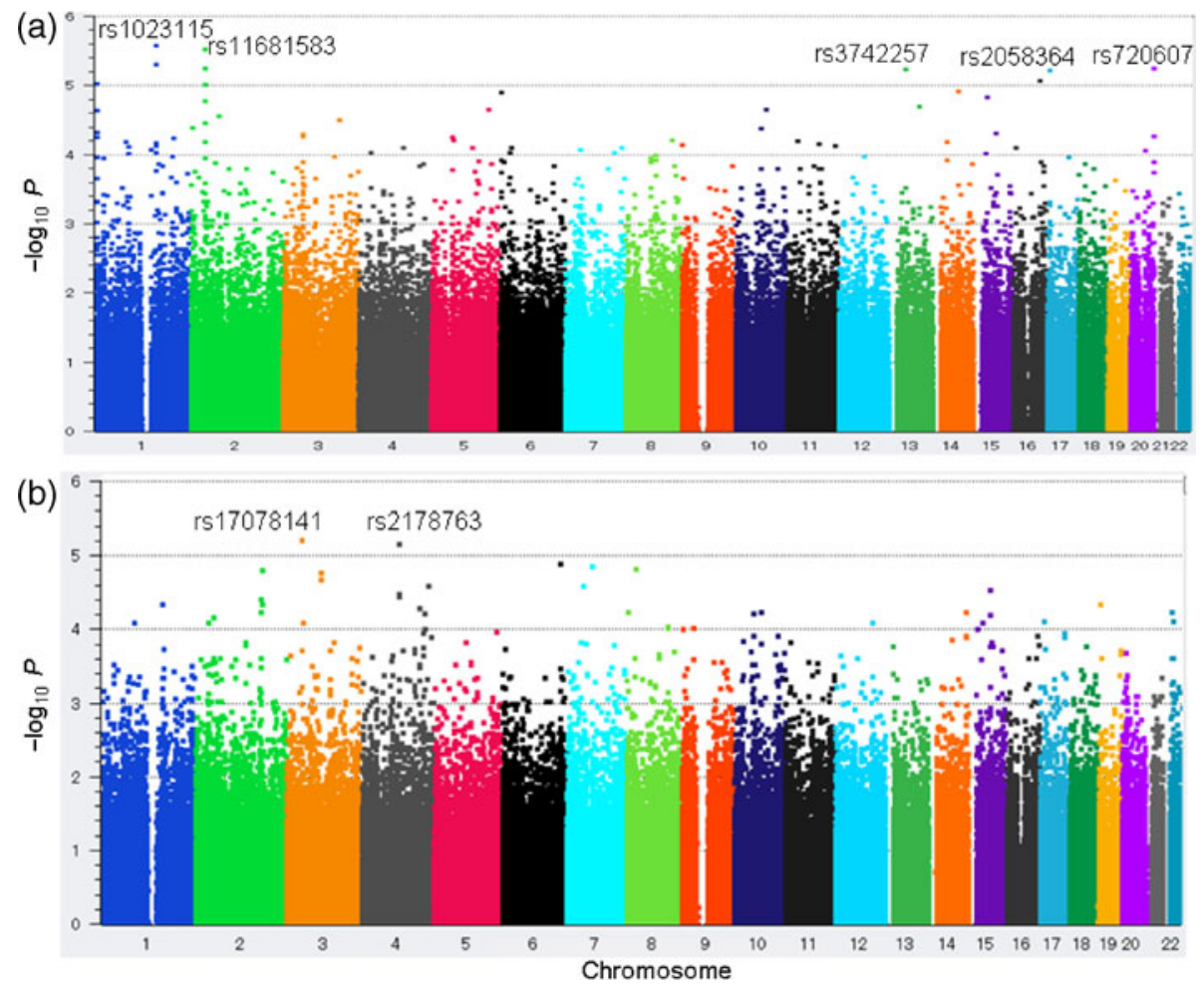

Figure 1. Manhattan plot of the genomewide $P$ values of association. The $-\log _{10} P$ values $(y$ axis) are presented against their chromosomal positions ( $x$-axis). The top significant associated SNPs are showed. A $P$-value threshold of $1 \times 10^{-5}$ was taken to represent suggestive association. (a) Analysis for case-control comparison and (b) analysis for case with and without cerebral infarction comparison.

\section{Association with ACTA2 and RNF213}

We investigated the association of SNPs in ACTA2 and $R N F 213$ with MMD (table 5 in electronic supplementary material). Neither association of SNPs in ACTA2 nor $R N F 213$ with MMD was confirmed. However, a cluster of 10 of 25 SNPs in $R N F 213$ gene region had $P$ values less than 0.05 , suggesting a possible association with $R N F 213$ with MMD.

\section{Discussion}

We conducted a GWAS to identify the major founder variant for Caucasian MMD but could not find any major founder variant in Caucasian MMD as we did in East Asian MMD. However, we found several suggestive association regions that might be responsible for the Caucasian MMD.

A major founder mutation of $\mathrm{p} . \mathrm{R} 4810 \mathrm{~K}$ in $R N F 213$ within $17 \mathrm{q} 25.3$ was first rigorously identified in East Asian MMD (Liu et al. 2011) and perfectly replicated in different cohorts (Miyatake et al. 2012; Wu et al. 2012). The number of patients with MMD was estimated to be at least 53,800 in East Asian populations (Liu et al. 2012). In contrast, we could not detect any founder variants in RNF213 for Caucasian MMD, suggesting the different genetic background between Caucasian and East Asian MMD. The low prevalence of Caucasian MMD might be due to the lack of the predominant founder variant. However, it should be addressed that there was a cluster of SNPs in $R N F 213$ region weakly associated with MMD, suggesting $R N F 213$ as a potential susceptibility gene for MMD even in Caucasian.

Mutations in ACTA2 were first identified in Caucasian MMD (Guo et al. 2009), but they have not been replicated in Japanese and another Caucasian MMD (Shimojima and Yamamoto 2009; Liu et al. 2011; Roder et al. 2011). We confirmed the absence of association. Importantly, caseonly association analysis revealed that MYRIP was located around the suggestive association region for cerebral infarction (figures $1 \mathrm{~b}$ and 2e). MYRIP has the highest expression in brain. Fukuda and Kuroda (2002) showed that the C-terminal domain of MYRIP directly bound actin, which was encoded by ACTA2. The above features suggest that MYRIP might be responsible for the progression of MMD in Caucasian population. This observation warrants further investigation in future. The present study, on the other hand, failed to demonstrate the association of SNPs in RNF213 (see table 5 in electronic supplementary material) with MMD.

This study had several limitations. First, the small number of available samples caused by the rareness of MMD might be the most critical limitation. In this relation, the current study suggests that our study assumption for calculation of statistical power is not realistic. If so, there may be no 

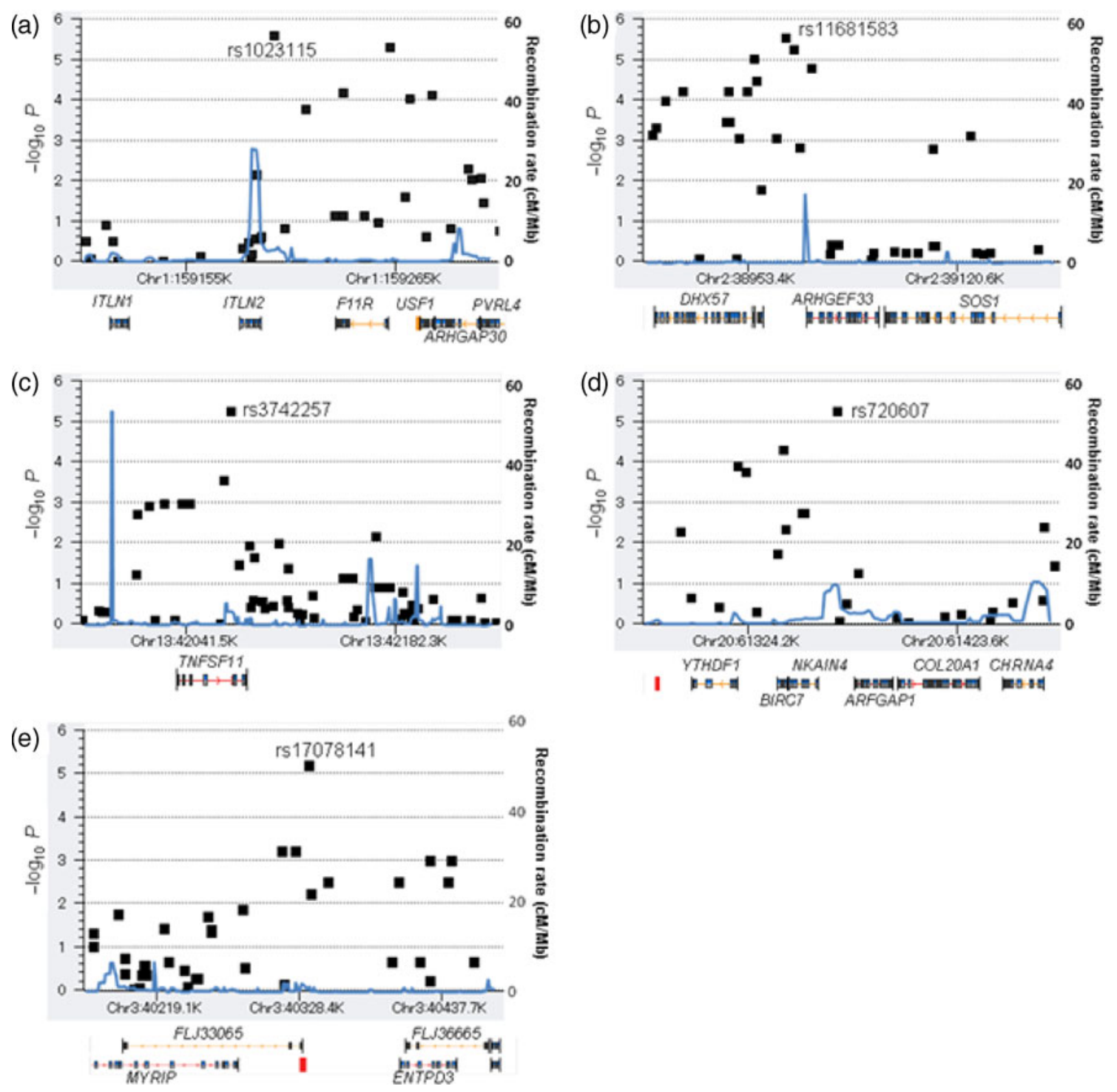

Figure 2. Regional association plots for the suggestive association regions. (a) Association plot for loci at rs 1023115 on 1q23.3. (b) Association plot for loci at rs11681583 on 2p22.1. (c) Association plot for loci at rs 720607 on $13 q 14.11$. (d) Association plot for loci at rs2058364 on 20q13.33. (e) Association plot for loci at rs17078141 on 3p22.1. The results $\left(-\log _{10} P\right)$ are for SNPs in the regions flanking the top SNPs. The recombination rate (blue line) was estimated based on the CEU, YRI and JPT+CHB populations from the Phase 2 HapMap (Release 22, NCBI 36). The annotated genes within the suggestive association regions are shown beneath the plots.

such predominant susceptible or causative rare variant in Caucasian cases as it is in East Asian MMD. Second, the rare variants cannot be discovered through GWAS. Nextgeneration sequencing will be of help in identifying rare variants. Third, several missense variants identified in candidate genes were unlikely to be causative genes because they are common in general Caucasian population. Searching for mutations by exome in a large number of Caucasian patients in these candidate genes is needed in future to find causative genes.

In summary, we were unsuccessful in our attempt to identify predominant founder variants in Caucasian MMD. The absence of the founder variant in Caucasians is in strong contrast to the major founder variant that has been identified in East Asians. Taken together, the present result probably indicates that locus heterogeneity as well as disease heterogeneity may be much greater in Caucasian MMD than in Asian MMD. Further study is warranted to investigate the genetic basis for global MMD.

\section{Acknowledgements}

This work was supported mainly by grants from the Ministry of Education, Culture, Sports, Science and Technology of Japan (Kiban Kenkyu A: 22249020) and the Ministry of Health, Labour and Welfare of Japan (H23-Nanji-Ippan-019, chaired by Dr Nobuo 
Hashimoto) and partially by a grant from Creative Scientific Research (19G50314). We thank all individuals for their participation in this study. We also thank Dr David Školoudík (Department of Neurology, University Hospital, Ostrava, Czech Republic); Dr Martin Kucharík (Department of Neurology, 1st Faculty of Medicine, Charles University and General Teaching Hospital, Prague, Czech Republic); Dr Aleš Tomek (Department of Neurology, 2nd Faculty of Medicine, Charles University and Motol University Hospital, Prague, Czech Republic); Dr Martin Kovár (Department of Neurology, Na Homolce Hospital, Prague, Czech Republic); Dr Jan Fiksa (Department of Neurology, Military University Hospital, Prague, Czech Republic); Dr Michael J. Schmeisser (Institute for Anatomy and Cell Biology, Ulm University, Ulm, Germany); Dr Vera Alexandra Neves dos Santos (Department of Pediatry, Hospital de Faro, Faro, Portugal); Dr Vera Peters (Department of Neurosurgery, University of Tübingen, Tübingen, Germany); Prof. Daniela Berg and Ms Claudia Schulte (both Department of Neurology, University of Tübingen, Tübingen, Germany) and Prof. Martin Schöning (Department of Pediatrics, University of Tübingen, Tübingen, Germany) for their patients recruitment.

\section{References}

Fukuda M. and Kuroda T. S. 2002 Slac2-c (synaptotagmin-like protein homologue lacking C2 domains-c), a novel linker protein that interacts with Rab27, myosin Va/VIIa, and actin. J. Biol. Chem. 277, 43096-43103.

Goto Y. and Yonekawa Y. 1992 Worldwide distribution of moyamoya disease. Neurol. Med. Chir. (Tokyo) 32, 883-886.

Guo D. C., Papke C. L., Tran-Fadulu V., Regalado E. S., Avidan N., Johnson R. J. et al. 2009 Mutations in smooth muscle alpha-actin (ACTA2) cause coronary artery disease, stroke, and Moyamoya disease, along with thoracic aortic disease. Am. J. Hum. Genet. 84, 617-627.

Kuroda S. and Houkin K. 2008 Moyamoya disease: current concepts and future perspectives. Lancet Neurol. 7, 1056-1066.

Liu W., Hashikata H., Inoue K., Matsuura N., Mineharu Y., Kobayashi H. et al. 2010 A rare Asian founder polymorphism of Raptor may explain the high prevalence of Moyamoya disease among East Asians and its low prevalence among Caucasians. Environ. Health Prev. Med. 15, 94-104.

Liu W., Morito D., Takashima S., Mineharu Y., Kobayashi H., Hitomi T. et al. 2011 Identification of RNF213 as a susceptibility gene for moyamoya disease and its possible role in vascular development. PLoS One 6, e22542.

Liu W., Harada K. H., Hitomi T., Kobayashi H. and Koizumi A. 2012 Distribution of moyamoya disease susceptibility polymorphism p.R4810K in RNF213 in East and Southeast Asian populations. Neurol. Med. Chir. (Tokyo) 52, 299-303.

Miyatake S., Miyake N., Touho H., Nishimura-Tadaki A., Kondo Y., Okada I. et al. 2012 Homozygous c. $14576 \mathrm{G}>\mathrm{A}$ variant of RNF213 predicts early-onset and severe form of moyamoya disease. Neurology 78, 803-810.

Roder C., Peters V., Kasuya H., Nishizawa T., Wakita S., Berg D. et al. 2011 Analysis of ACTA2 in European Moyamoya disease patients. Eur. J. Paediatr. Neurol. 15, 117-122.

Shimojima K. and Yamamoto T. 2009 ACTA2 is not a major disease-causing gene for moyamoya disease. J. Hum. Genet. 54, 687-688.

Suzuki J. and Takaku A. 1969 Cerebrovascular "moyamoya" disease. Disease showing abnormal net-like vessels in base of brain. Arch. Neurol. 20, 288-299.

Takeuchi K. and Shimizu K. 1957 Hypogenesis of bilateral internal carotid arteries. Brain Nerve 9, 37-43.

Wu Z., Jiang H., Zhang L., Xu X., Zhang X., Kang Z. et al. 2012 Molecular analysis of RNF213 gene for moyamoya disease in the Chinese Han population. PLoS One 7, e48179. 